\title{
Clinical features and determinants of COPD exacerbation in the Hokkaido COPD cohort study
}

\author{
Masaru Suzuki ${ }^{1}$, Hironi Makita' ${ }^{1}$ Yoichi M. Ito², Katsura Nagai ${ }^{1}$, Satoshi Konno ${ }^{1}$ and \\ Masaharu Nishimura' for the Hokkaido COPD Cohort Study Investigators
}

Affiliations: ${ }^{1}$ First Dept of Medicine, Hokkaido University School of Medicine, Sapporo, ${ }^{2}$ Dept of Biostatistics, Hokkaido University Graduate School of Medicine, Sapporo, Japan.

Correspondence: M. Nishimura, First Dept of Medicine, Hokkaido University School of Medicine, North 15 West 7, Kita-ku, Sapporo 060-8638, Japan. E-mail: ma-nishiamed.hokudai.ac.jp

ABSTRACT Exacerbations are among the major factors that may affect the natural history of chronic obstructive pulmonary disease (COPD). The aim was to investigate the clinical characteristics and determinants of COPD exacerbations in our 5-year observational cohort study which had a very low exacerbation frequency.

A total of 279 patients with COPD participated in the Hokkaido COPD cohort study, and 268 subjects who had clinical data for multiple visits were analysed. Exacerbation was defined in multiple ways: the patient's subjective complaint, symptom definition, requiring prescription change, requiring antibiotic treatment, or requiring hospital admission.

Exacerbation frequency (events per person per year) was $0.78 \pm 1.16,0.24 \pm 0.47,0.20 \pm 0.43,0.13 \pm 0.28$ and $0.06 \pm 0.19$ for subjective complaint and symptom, prescription, antibiotic and hospital admission definitions, respectively. Exacerbation events did not significantly affect the annual decline in forced expiratory volume in $1 \mathrm{~s}$. A high St George's Respiratory Questionnaire total score, especially activity score, and a low body mass index were strongly associated with exacerbation-free survival, exacerbation frequency and development of recurrent exacerbations.

Despite the low exacerbation frequency in our cohort, impaired health-related quality of life and weight loss were found to be independent risk factors for COPD exacerbations.

@ERSpublications

Impaired health-related quality of life and weight loss are independent risk factors for COPD exacerbations http://ow.ly/qQRAG

For editorial comments see page 1220 .

This article has supplementary material available from www.erj.ersjournals.com

Received: June 282013 | Accepted after revision: Oct 272013 | First published online: Nov 142013

Support statement: This study was supported by a scientific research grant to the Hokkaido COPD Cohort Study from the Ministry of Education, Science, Culture and Sports of Japan (17390239 and 2139053 to M. Nishimura), Nippon Boehringer Ingelheim (Tokyo, Japan), Pfizer Inc. (Tokyo, Japan) and a grant to the Respiratory Failure Research Group H23-nanchi-ippan-024) from the Ministry of Health, Labor and Welfare, Japan.

Conflict of interest: Disclosures can be found alongside the online version of this article at www.erj.ersjournals.com Copyright $\odot$ ERS 2014 


\section{Introduction}

Chronic obstructive pulmonary disease (COPD) is characterised by persistent airflow limitation that is usually progressive, and is a leading cause of morbidity and mortality worldwide [1]. Exacerbations of COPD are an acute event characterised by a worsening of respiratory symptoms, and are very important in the clinical course of COPD as they are associated with poor quality of life (QoL), increased mortality and high socioeconomic costs [1-3]. Recently, HURST et al. [4] reported that patients who have frequent exacerbations belong to a clinically stable phenotype that is susceptible to further exacerbations. Therefore, it would be critical to determine the clinical characteristics and predictors of exacerbations for better management of COPD patients.

The Hokkaido COPD cohort study is a carefully designed, multicentre, observational cohort that primarily aims to examine the annual decline in forced expiratory volume in $1 \mathrm{~s}(\mathrm{FEV} 1)$ over a period of 5 years based on clinical phenotypes in patients with smoking-related COPD $[5,6]$. We have already reported that the rate of annual change in FEV1 was highly variable among patients with COPD and was not associated with exacerbation frequency [6]. A unique finding of our cohort study was that the exacerbation frequency was much lower than the previous large-scale clinical trials [6]. However, the characteristics and risk of exacerbations in a population with such low exacerbation frequency have not yet been clarified. In this study, the clinical characteristics and determinants of COPD exacerbations were examined in our 5-year observational cohort.

\section{Methods \\ Participants}

The recruitment of the COPD patients has been described previously [5, 6]. Briefly, 330 subjects with respiratory physician-diagnosed COPD were recruited at Hokkaido University Hospital (Sapporo, Japan) and nine affiliated hospitals from May 2003 to May 2005. All were aged $\geqslant 40$ years and were either current or former smokers with a smoking history of at least 10 pack-years. Subjects with clinically diagnosed asthma were excluded. 30 subjects were excluded due to consent withdrawal, or were ineligible for inclusion before visit 1, and a total of 300 subjects were followed. During the first year of follow-up, the diagnosis of COPD was reconfirmed in 279 subjects based on the spirometric criteria of the Global Initiative for Chronic Obstructive Lung Disease (GOLD) guidelines (post-bronchodilator FEV1/forced vital capacity <0.70) [1], and the subjects were eligible for subsequent follow-up. In this study, 268 subjects (26\% GOLD 1, 45\% GOLD 2, 24\% GOLD 3 and 5\% GOLD 4) who had clinical data for multiple visits were analysed. Of the 268 subjects, $184(69 \%)$ completed a 5-year follow-up period. The reasons for dropout from the initial 279 subjects have been described previously [6]. The median follow-up period was 4.97 years. Characteristics of subjects classified by severity of airflow limitation are shown in table 1 . The Ethics Committee of Hokkaido University School of Medicine approved the study protocol, and written, informed consent was obtained from all participants.

\section{Study protocol}

The details of the study protocol for the Hokkaido COPD cohort study have been described elsewhere $[5,6]$. Most subjects, except for those in GOLD 1, visited outpatient clinics at each hospital monthly or bimonthly for regular clinical check-ups (table S1). On the first visit, demographic information, including sex, age, height, weight, smoking history, medical history and medications, and information on pulmonary symptoms were collected. Every 6 months any changes in smoking status, medical history and pharmacotherapy were monitored. Health-related QoL was assessed by St George's Respiratory Questionnaire (SGRQ) [7] every year, and blood was also sampled every year for measurements of circulating blood cell counts, serum IgE and C-reactive protein. Spirometry was performed before and after inhalation of a bronchodilator on every visit. Chest computed tomography (CT) scans were performed in the supine position with breath held at full inspiration. Severity of emphysema was visually assessed by three independent pulmonologists according to the modified Goddard scoring system $[5,6,8]$.

\section{Assessment of exacerbation}

In order to collect exacerbation information, prepaid reply postcards were sent each month to all participants. Replies were received from almost all participants (reply rate $>99 \%$ ). The questionnaire items on the postcard are described in the online supplementary material. If exacerbation was suspected, information was always reconfirmed by telephone interview and/or by the medical charts of subjects when they visited the clinic. In addition, the subjects' medical records were periodically checked, and attending physicians were asked about the subjects' conditions when necessary.

Exacerbation of COPD was defined in the following ways: 1) patient's subjective complaint by prepaid reply postcard (any clinical symptoms that did not meet symptom definition criteria); 2) worsening or new onset 
TABLE 1 Characteristics of subjects classified by severity of airflow limitation

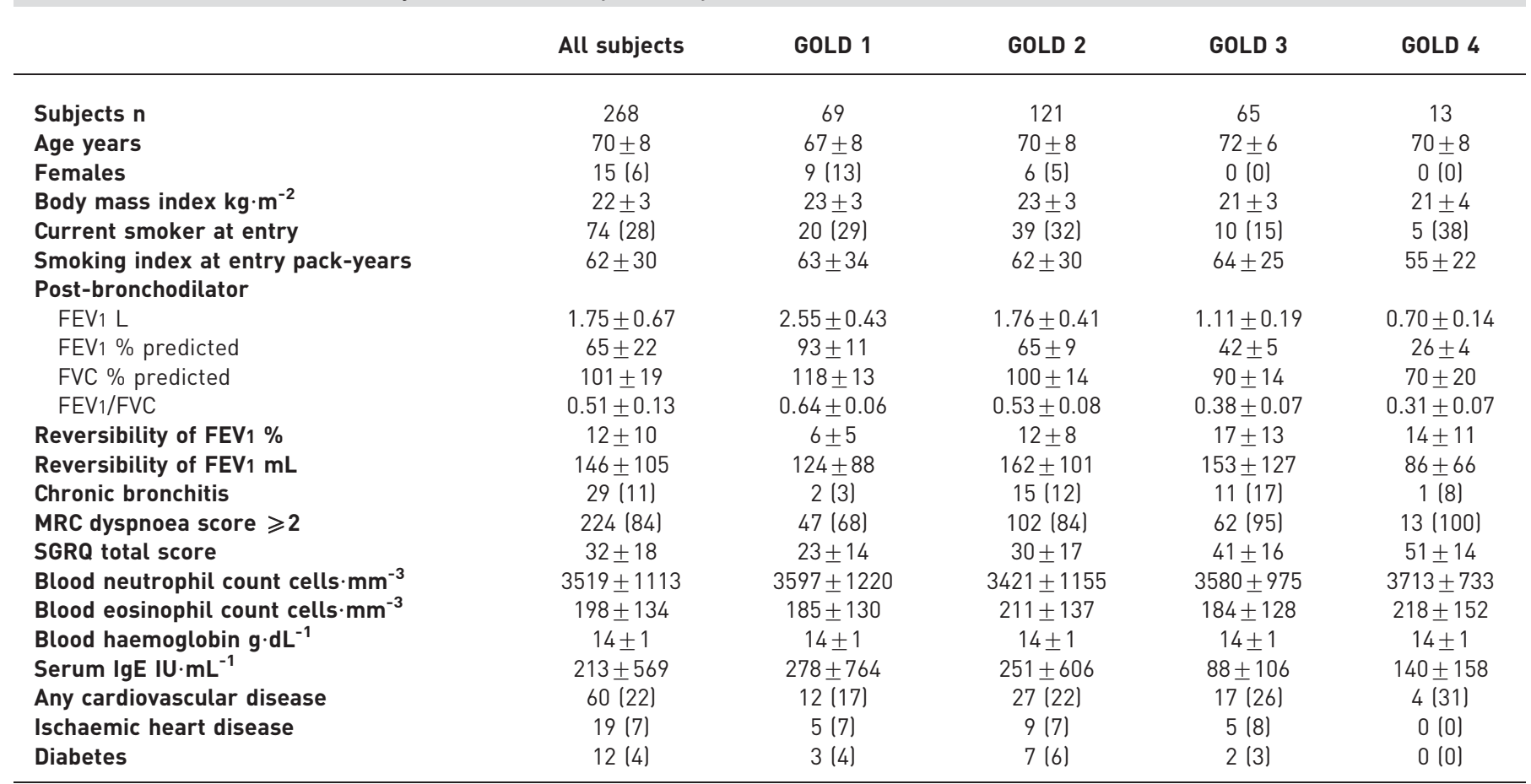

Data are presented as mean \pm SD or $\mathrm{n}(\%)$, unless otherwise stated. GOLD: Global Initiative for Chronic Obstructive Lung Disease; FEV1: forced expiratory volume in $1 \mathrm{~s}$; FVC: forced vital capacity; MRC: Medical Research Council; SGRQ: St George's Respiratory Questionnaire.

of either two major symptoms (increased dyspnoea, change in sputum purulence or increased sputum volume) or any one major symptom plus any minor symptoms (fever, increased cough or wheezing) compared with baseline (symptom definition); 3) symptom criteria plus requiring prescription change (prescription definition); 4) symptom criteria plus antibiotic treatment (antibiotic definition); and 5) symptom criteria plus hospital admission (admission definition). Radiologically proven pneumonia was not excluded from exacerbation events as many patients with severe exacerbation are examined by CT scan in Japan and bronchopneumonia is often detected even if chest radiography is almost normal.

\section{Statistical analysis}

Details of statistical analysis are available in the online supplementary material. Differences among the groups were analysed using the t-test, the Mann-Whitney U-test or the Chi-squared test, where appropriate. Bivariate correlations were analysed using Spearman's rank correlation coefficient. Factors associated with exacerbation-free survival were analysed using a Cox proportional hazards model and the Kaplan-Meier method with the log-rank test. Factors associated with exacerbation frequency were analysed using a Poisson regression model. Factors associated with recurrent exacerbation were analysed using the Prentice, Williams and Peterson (PWP) total time model that is based on the Cox proportional hazards model for recurrent event data [9]. Here, the total time refers to the time interval from time origin 0 to the occurrence of each event. Significant variables in univariate models were included simultaneously in a multivariate model. Statistical significance was defined as $\mathrm{p}<0.05$.

\section{Results}

The cumulative number of exacerbation events and the number of subjects who experienced exacerbations during the follow-up period differed depending on the definition criteria (fig. 1). Indeed, the number of exacerbation events became much lower compared to the number of patients' subjective complaints when symptoms were carefully confirmed. There were $16(6.6 \%)$ out of 243 exacerbation events that were not picked up by subjective complaint in the postcard but by confirmation of symptoms and prescription changes by interview and/or medical records. COPD exacerbation frequency (events per person per year) during the follow-up period was $0.78 \pm 1.16,0.24 \pm 0.47,0.20 \pm 0.43,0.13 \pm 0.28$ and $0.06 \pm 0.19$ for subjective complaint and symptom, prescription, antibiotic and admission definitions, respectively. The exacerbation frequency was higher in subjects with severe airflow limitation (GOLD 3-4) than in those with 


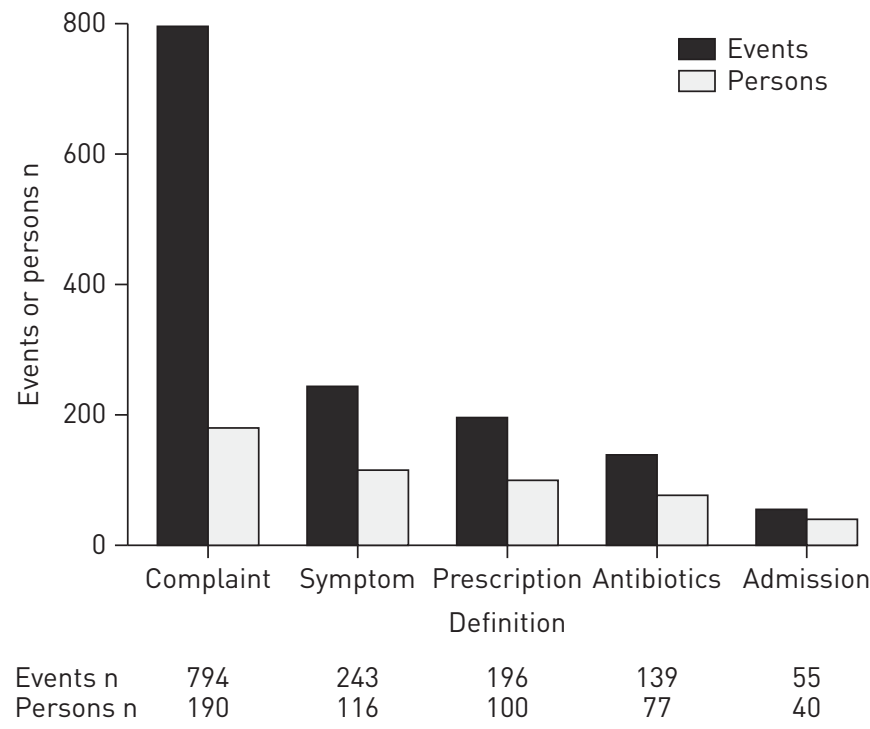

FIGURE 1 The number of exacerbation events or persons during the follow-up period. Exacerbation was defined by patient's subjective complaints and the symptom, prescription, antibiotic and admission definitions.

mild airflow limitation (GOLD 1-2) (table 2). There were very few subjects who experienced $\geqslant 2$ exacerbations per year (only three subjects by symptom or prescription definitions and none of the subjects by antibiotics or admission definitions). Subjects who experienced at least one exacerbation during the follow-up period had lower lung function, more dyspnoea and higher SGRQ total score (i.e. impaired health-related QoL) compared to subjects who had no exacerbation (table S2). Among subjects who experienced at least one exacerbation, $50 \%$ of subjects by symptom definition, $48 \%$ by prescription definition, $42 \%$ by antibiotics definition and $25 \%$ by admission definition developed multiple exacerbation events during the follow-up period (recurrent exacerbation). The number of exacerbation events was higher in the spring (March to June) and autumn (October to November) (fig. 2).

Subjects who experienced exacerbations within the first year of follow-up had more frequent exacerbations after the first year of follow-up (fig. 3), which confirmed the recurrent nature of COPD exacerbations. However, the annual decline in FEV1 was not affected by exacerbation, regardless of its definition and the degree of airflow limitation (fig. S1), whereas subjects who experienced more than one exacerbation defined by admission criteria per year tended to show a more rapid FEV1 decline compared to subjects with less exacerbations $(\mathrm{p}=0.07)$ (fig. S2). There was no significant correlation between the annual decline in FEV1 and exacerbation frequency at any definition (data not shown).

A multivariate Cox proportional hazards model showed that low body mass index (BMI) and high SGRQ total score were significant and independent predictors for the early development of the first exacerbation event defined as both prescription change and hospital admission (table 3 and tables S3 and S4), and Kaplan-Meier curves for the classification groups by BMI and SGRQ total score were clearly separated (fig. 4).

The multivariate Poisson regression model showed that low BMI, high SGRQ total score, low FEV1 and low haemoglobin were significantly associated with exacerbation frequency defined as prescription change, and low BMI and high SGRQ total score were significantly associated with exacerbations defined as hospital

TABLE 2 Exacerbation frequency classified by severity of airflow limitation

\begin{tabular}{|c|c|c|c|c|c|}
\hline Subjects & 268 & 69 & 121 & 65 & 13 \\
\hline \multicolumn{6}{|c|}{ Exacerbation events per person per year } \\
\hline Subjective complaint & $0.78 \pm 1.16$ & $0.58 \pm 0.79$ & $0.63 \pm 0.94$ & $1.09 \pm 1.51^{*, \#}$ & $1.80 \pm 1.86^{*, \#}$ \\
\hline Symptom definition & $0.24 \pm 0.47$ & $0.16 \pm 0.29$ & $0.16 \pm 0.27$ & $0.37 \pm 0.57^{*, \#}$ & $0.81 \pm 1.15^{*, \#}$ \\
\hline Prescription definition & $0.20 \pm 0.43$ & $0.12 \pm 0.26$ & $0.14 \pm 0.25$ & $0.30 \pm 0.49^{*}, \#$ & $0.77 \pm 1.13^{*, \#, \oplus}$ \\
\hline Antibiotics definition & $0.13 \pm 0.28$ & $0.09 \pm 0.23$ & $0.09 \pm 0.22$ & $0.20 \pm 0.35^{*, \#}$ & 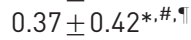 \\
\hline Admission definition & $0.06 \pm 0.19$ & $0.01 \pm 0.03$ & $0.06 \pm 0.19 *$ & $0.10 \pm 0.27^{*}, \#$ & $0.09 \pm 0.15^{*}$ \\
\hline
\end{tabular}

Data are presented as $n$ or mean \pm SD. GOLD: Global Initiative for Chronic Obstructive Lung Disease. *: $p<0.05$ versus GOLD 1; ${ }^{\#}$ p $<0.05$ versus GOLD 2; ${ }^{\circ}: \mathrm{p}<0.05$ versus GOLD 3. 
FIGURE 2 The number of exacerbation events in each month during the followup period. Exacerbation was defined by the symptom, prescription, antibiotic and admission definition.

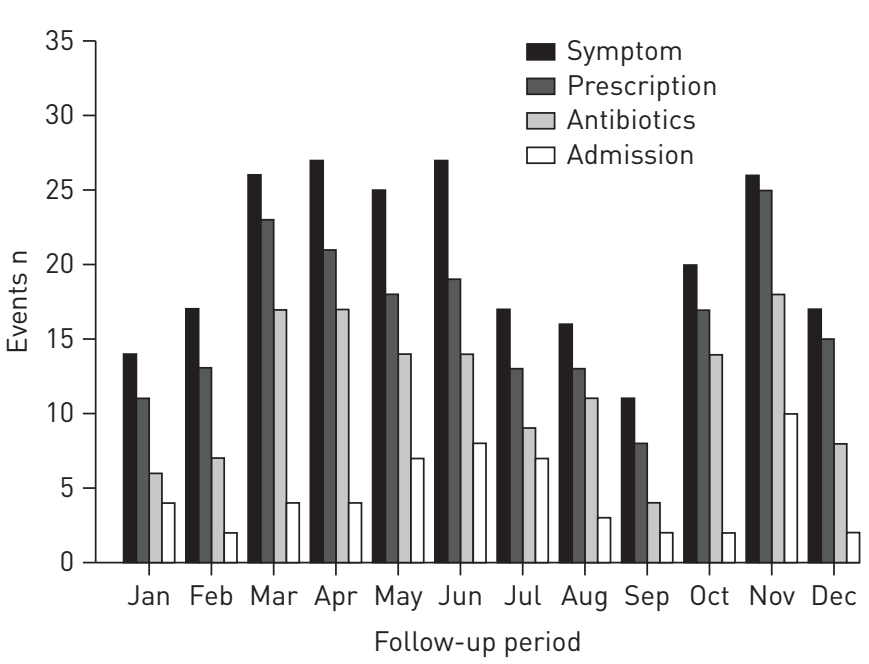

admission (table 3 and tables S5 and S6). Furthermore, the multivariate PWP total time model showed that high SGRQ total score and low haemoglobin were significant predictors for the development of recurrent exacerbations defined as prescription change, and that older age, low BMI and high SGRQ total score were significant predictors for the development of recurrent exacerbations defined as hospital admission (table 3 and tables S7 and S8).

Since a high SGRQ total score was significantly associated with all of exacerbation-free survival, exacerbation frequency and the development of recurrent exacerbations, the SGRQ domain scores of symptoms, activity, and impact were also assessed. The SGRQ activity score was found to be the only domain that was significantly associated with all of the above analyses (table S9).

\section{Discussion}

In this study, the intention was to clarify the clinical characteristics and determinants of COPD exacerbations using the Hokkaido COPD cohort study population. The strongest point of this cohort study was that it was very carefully designed and performed, thus making it possible to collect accurate information regarding each patient's complaints, symptoms and clinical data during COPD exacerbations. Although a COPD exacerbation is defined in the GOLD guidelines as an acute event characterised by a worsening of the patient's respiratory symptoms and leads to a change in medication [1], a general definition for COPD exacerbation has not been accepted. Moreover, several levels regarding the severity of exacerbations are required. Therefore, COPD exacerbation was defined in multiple ways in the present study. It was found that the number of exacerbation events and the number of subjects who experienced exacerbations were very different depending on the definition criteria, especially between patients' subjective complaints and confirmed symptoms. Importantly, the same patients seemed to repeatedly complain about their poor physical condition even if they did not have enough respiratory symptoms, since the number of exacerbation events was less than the number of subjects who experienced exacerbation events when the symptom definition was applied (fig. 1). Therefore, it is very important for physicians to confirm patients' respiratory symptoms carefully for the diagnosis of COPD exacerbation. However, it is possible that we missed some symptomatic events even though the symptom information was reconfirmed by telephone interview and/or by the medical charts. It may also have been possible that some subjects were reluctant to declare their symptoms accurately to medical staff, despite the fact that they reported the symptoms on the postcards. Furthermore, some subjects might not have been willing to complain in the postcards so we might have missed actual exacerbations. We think that enhancement and encouragement of reporting using more sensitive tools such as a daily diary or an electronic personal digital assistant would be ideal for more accurate symptom assessment.

A unique finding of this study is the much lower exacerbation frequency during the study period compared to recent large-scale clinical studies, such as TORCH [10], UPLIFT [11] and ECLIPSE [4, 12]. Importantly, the present population included patients with mild airflow limitation (GOLD 1), unlike the previously mentioned clinical studies that did not recruit GOLD 1 patients. It was confirmed that exacerbations became more frequent as the severity of airflow limitation increased (table 2), which was consistent with previous studies $[4,13,14]$, suggesting that recruitment of patients with milder airflow limitation may contribute to the lower exacerbation frequency. However, the exacerbation frequency in the present study 

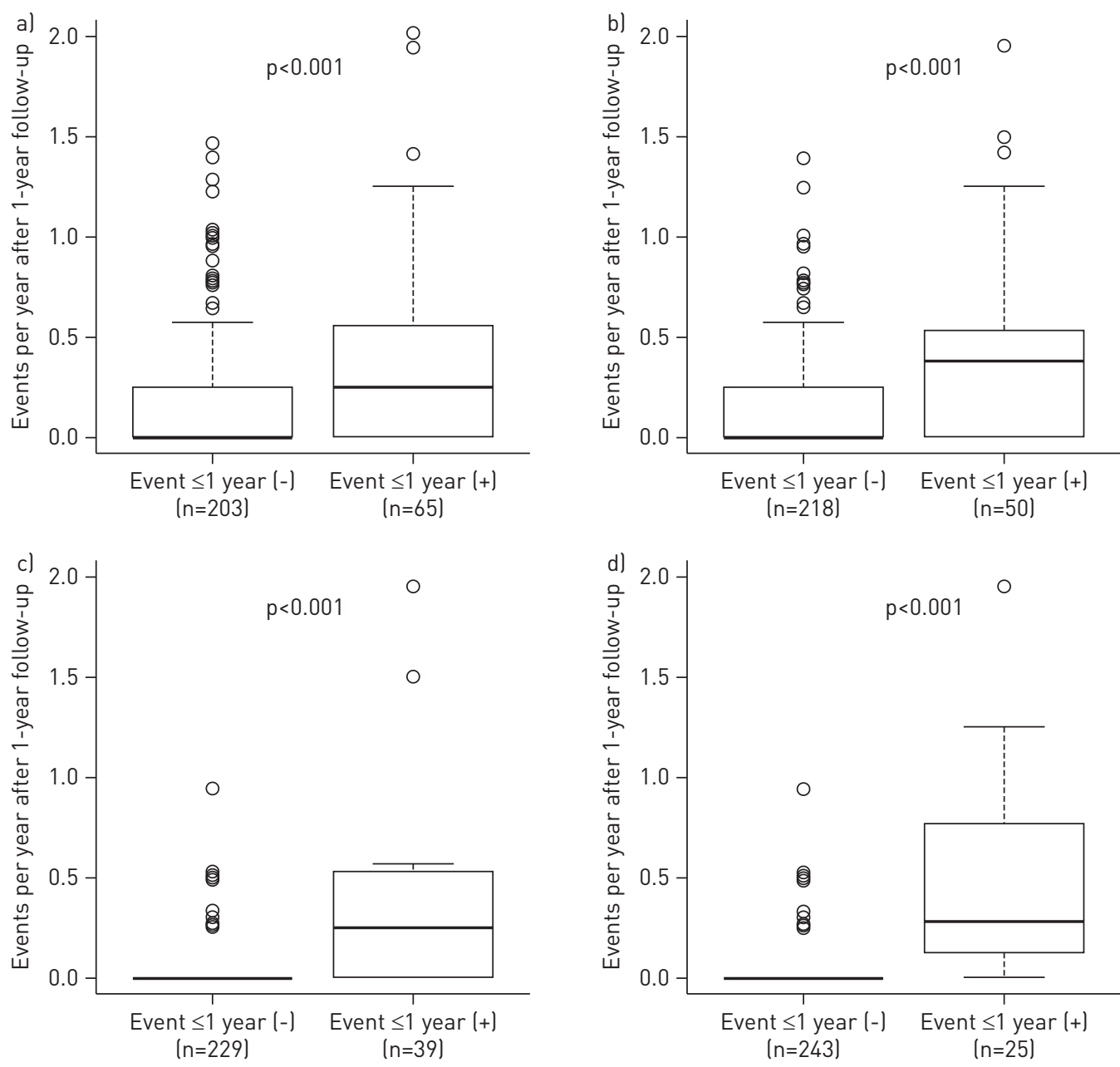

FIGURE 3 Box plots of the exacerbation frequency after the first year of follow-up. Exacerbation is defined by a) symptom definition, b) prescription definition, c) antibiotic definition and d) the admission definition. Subjects were divided into two groups: subjects who did not experience exacerbations within the first year of follow-up (event $\leqslant 1$ year $(-))$ and subjects who experienced exacerbations within the first year of follow-up (event $\leqslant 1$ year $(+)$ ).

was still lower than that of previous studies even when compared with patients with the same severity of airflow limitation. Specifically, the mean frequency of exacerbations (events per person per year), defined as prescription change in each GOLD category, was $0.14,0.30$ and 0.77 for GOLD 2, GOLD 3 and GOLD 4, respectively, in the present study, whereas the mean was $0.7-0.9,1.1-1.3$ and 1.2-2.0 for GOLD 2, GOLD 3 and GOLD 4, respectively, in previous large-scale clinical studies. Similarly, the mean frequency of hospital admission was $0.06,0.10$ and 0.09 for GOLD 2, GOLD 3 and GOLD 4, respectively, in the present study, whereas the mean was $0.11-0.2,0.25-0.3$ and $0.4-0.54$ for GOLD 2, GOLD 3 and GOLD 4, respectively, in previous studies $[1,4,10,12]$. Such a lower frequency of exacerbation was also observed in the subgroup analysis of the Japanese patients participating the UPLIFT study and in another Japanese report $[15,16]$. The frequency of chronic bronchitis in our cohort was also lower compared to the other studies [12, 17]. Thus, national characteristics, such as the healthcare system and socioeconomic status, may affect the discrepancy in the frequency of exacerbations and chronic bronchitis between Japan and the other geographical regions. Another possible reason would be a selection bias in our cohort study, since all of the subjects were recruited and treated by respiratory specialists at a university hospital and its affiliated hospitals. Even though the exacerbation frequency was low in the present study, the recurrent nature of exacerbations was confirmed by showing that subjects who experienced exacerbations within the first year of follow-up experienced more frequent exacerbations after the first year of follow-up (fig. 3 ).

It was found that the number of exacerbation events was higher in the spring and autumn months, but not in the winter (fig. 2), which was an unexpected finding because COPD exacerbations were reported to be more frequent in the winter months $[18,19]$. Our cohort study was performed in the north of Japan, where the winter is very cold and accompanied by significant snowfall; therefore, patients with COPD may tend to 
TABLE 3 Significant factors related to chronic obstructive pulmonary disease exacerbation in the multivariable models

Admission definition

\begin{tabular}{|c|c|c|c|c|}
\hline & & \multirow[b]{2}{*}{ Hazard ratio $(95 \% \mathrm{CI})$} & \multirow[b]{2}{*}{ p-value } \\
\hline & Hazard ratio $(95 \% \mathrm{Cl})$ & p-value & & \\
\hline \multicolumn{5}{|c|}{ Factors related to exacerbation-free survival ${ }^{\#}$} \\
\hline $\mathrm{BMI} 1 \mathrm{~kg} \cdot \mathrm{m}^{-2}$ increase & $0.93(0.87-0.99)$ & 0.03 & $0.86(0.76-0.96)$ & 0.006 \\
\hline SGRQ total score 4 points increase & $1.12(1.06-1.19)$ & $<0.001$ & $1.19(1.08-1.30)$ & $<0.001$ \\
\hline \multicolumn{5}{|c|}{ Factor relating to exacerbation frequency ${ }^{\pi}$} \\
\hline BMI $1 \mathrm{~kg} \cdot \mathrm{m}^{-2}$ increase & $0.95(0.90-0.99)$ & 0.03 & $0.87(0.79-0.95)$ & 0.004 \\
\hline SGRQ total score 4 points increase & $1.09(1.05-1.14)$ & $<0.001$ & $1.11(1.03-1.20)$ & 0.008 \\
\hline \multicolumn{5}{|c|}{ Factors related to recurrent exacerbation ${ }^{+}$} \\
\hline Age 10 -year increase & & & $1.57(1.00-2.46)$ & 0.049 \\
\hline BMI $1 \mathrm{~kg} \cdot \mathrm{m}^{-2}$ increase & & & $0.88(0.80-0.98)$ & 0.02 \\
\hline SGRQ total score 4 points increase & $1.07(1.03-1.11)$ & $<0.001$ & $1.14(1.04-1.24)$ & 0.005 \\
\hline Haemoglobin $1 \mathrm{~g} \cdot \mathrm{dL}^{-1}$ increase & $0.87(0.78-0.97)$ & 0.02 & & \\
\hline
\end{tabular}

stay inside their homes. Since the trigger for a large number of exacerbations is a respiratory virus infection [20], such patients may have a lower chance of getting a viral infection in the community in the winter. Whatever the reason, the present result indicates that the seasonality of COPD exacerbations can vary depending on where the patients live due to climate differences.

Another notable finding of the present study is that whether subjects experienced exacerbation events or not during the follow-up period did not affect the annual decline in FEV1, regardless of its definition and the degree of airflow limitation (fig. 4). People may consider that this is an unexpected finding since it has been emphasised that COPD exacerbations accelerate the rate of decline in lung function. The GOLD guideline [1] cited two references for this statement $[21,22]$. However, using the Lung Health Study data, KANNER et al. [21] reported that lower respiratory tract illness promoted FEV1 decline in current smokers with mild COPD, but not in ex-smokers. Furthermore, DonALDSON et al. [22] showed a faster annual decline in FEV1 in patients with frequent exacerbations defined by symptom-based criteria $(>2.92$ events per person per year) when compared to patients with infrequent exacerbations $(<2.92$ events per person per year). In the present study, $85.1 \%$ of the subjects quit smoking during the follow-up period, and the exacerbation frequency was very low; thus, it is reasonable that the effect of exacerbation events on the annual decline in FEV1 in the present study was small. Moreover, the relationship between exacerbation events and a decline in FEV1 does not seem to be simple, since the large-scale UPLIFT study failed to show an improvement in the FEV1 decline, whereas it did show a significant reduction in the development of exacerbations by drug intervention [11]. However, there was also a tendency of rapid decline in FEV1 in subjects who experienced more than one exacerbation defined by admission criteria per year in the present study (fig. S2). Therefore, the effect of exacerbations on respiratory function seems to be larger in patients who experience frequent and more severe exacerbations.

In the multivariate analysis, impaired health-related QoL was significantly associated with exacerbation frequency (table 3), which is in line with previous studies [2, 4]. The present data extend this observation by showing that impaired health-related QoL was also strongly related to shorter exacerbation-free survival and the development of recurrent exacerbations defined as either prescription change or hospital admission (table 3). Furthermore, the SGRQ activity score, which is closely related to the dyspnoea scale and the 6-min walking distance [7], was the only domain that was significantly associated with exacerbation-free survival, exacerbation frequency and development of recurrent exacerbations (table S9). One explanation of the association between dyspnoea and exacerbations may come from the fact that dyspnoea is one of the major symptoms in the symptom definition. It is also possible that reduced activity and poor QoL are just confounders for other factors. However, there are several speculations regarding reduced activity with dyspnoea is a risk factor for exacerbations. First, mucus hypersecretion in subjects with dyspnoea may contribute to an increased risk of pulmonary infection that is an important trigger of COPD exacerbation. Secondly, lung hyperinflation in subjects with dyspnoea increases the imbalance of the ventilation/perfusion ratio and may be more susceptible to triggers of exacerbation [23]. It was also found that low BMI was 
BMI $<18.5$ and $S G R Q$ total score $\leq 30(n=18)$

-..- $\mathrm{BMI}<18.5$ and SGRQ total score $>30(\mathrm{n}=22)$

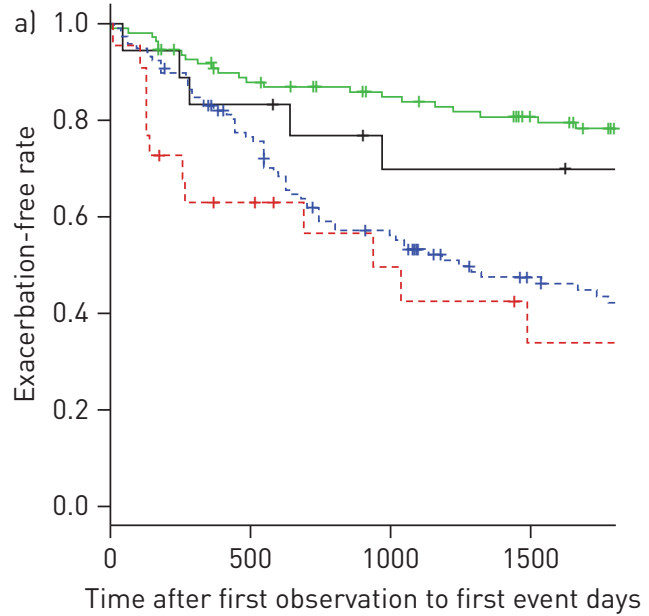

$\mathrm{BMI} \geq 18.5$ and $\mathrm{SGRQ}$ total score $\leq 30(\mathrm{n}=110)$
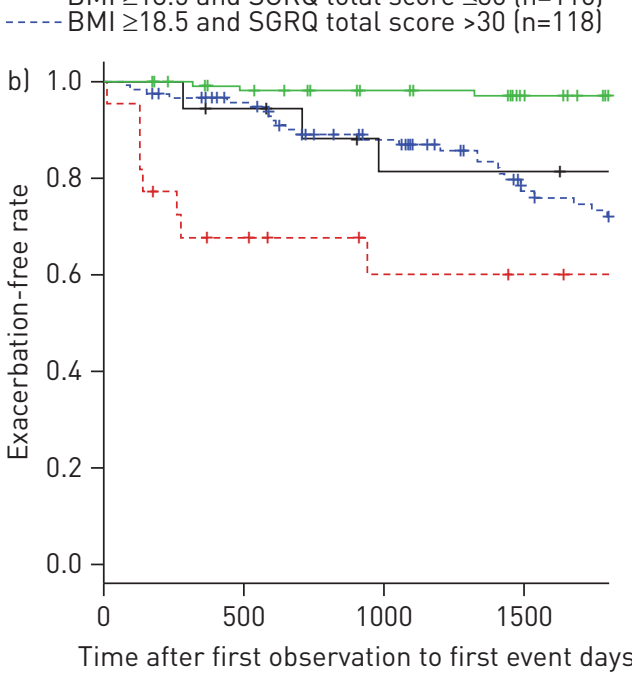

FIGURE 4 Kaplan-Meier curves for exacerbation-free survival. Exacerbations are defined by a) the prescription definition and b) the admission definition. Subjects were divided into four groups according to St George's Respiratory Questionnaire (SGRQ) total score and body mass index (BMI). Log rank p-value $<0.001$.

independently associated with COPD exacerbation (table 3). Poor nutritional status or low BMI has been shown to be associated with increased morbidity and mortality in the natural course of COPD and in patients hospitalised with COPD exacerbation [24, 25]. Therefore, it would be very important to identify patients who have limited physical activities due to dyspnoea or weight loss and perform a therapeutic intervention for such patients by medication, rehabilitation and supporting nutrition in order to reduce the morbidity and mortality from COPD exacerbations.

Although this study was a prospective, observational cohort study, it had several limitations. First, information about exacerbation history before study entry was not obtained. Since it was shown that the best predictor of exacerbations was a history of exacerbations [4], collecting exacerbation history would be important for the clinical management of patients with COPD. Regarding this point, the recurrent nature of exacerbations was confirmed using our prospective data (fig. 3). Secondly, most subjects were males, and there were no female patients in GOLD 3 and 4 categories. Therefore, the present findings may not simply be applied to female patients with COPD. Thirdly, we were unable to collect accurate information on anxiety and depression, which have been reported to be associated with COPD exacerbations [26, 27]. Finally, the sample size in this study was not as large as previous large-scale clinical studies.

In summary, the clinical characteristics and determinants of COPD exacerbations were identified in the Hokkaido COPD cohort study. In the present population, the exacerbation frequency was very low, while exacerbations appeared to be recurrent. Exacerbation events did not affect the annual decline in FEV1. Furthermore, poor health-related QoL and weight loss were strong predictors of the development of COPD exacerbations. Identification of patients at high risk for the development of exacerbations and appropriate intervention for such patients are crucial for the prevention of COPD exacerbations.

\section{Acknowledgements}

We would like to thank H. Ashikaga, A. Kondo and Y. Takagi at the Central Office of the Hokkaido COPD Cohort Study (Sapporo, Japan), and the medical doctors, nurses and technicians in all the hospitals involved in the study.

\section{References}

Global Initiative for Chronic Obstructive Lung Disease (GOLD). Global Strategy for the Diagnosis, Management, and Prevention of COPD. Updated 2013. www.goldcopd.org Date last updated: February 20, 2013. Date last accessed: June 25, 2013.

2 Seemungal TA, Donaldson GC, Paul EA, et al. Effect of exacerbation on quality of life in patients with chronic obstructive pulmonary disease. Am J Respir Crit Care Med 1998; 157: 1418-1422.

3 Soler-Cataluña JJ, Martínez-García MA, Román Sánchez P, et al. Severe acute exacerbations and mortality in patients with chronic obstructive pulmonary disease. Thorax 2005; 60: 925-931.

4 Hurst JR, Vestbo J, Anzueto A, et al. Susceptibility to exacerbation in chronic obstructive pulmonary disease. N Engl J Med 2010; 363: 1128-1138.

5 Makita H, Nasuhara Y, Nagai K, et al. Characterisation of phenotypes based on severity of emphysema in chronic obstructive pulmonary disease. Thorax 2007; 62: 932-937. 
Nishimura M, Makita H, Nagai K, et al. Annual change in pulmonary function and clinical phenotype in chronic obstructive pulmonary disease. Am J Respir Crit Care Med 2012; 185: 44-52.

7 Jones PW, Quirk FH, Baveystock CM, et al. A self-complete measure of health status for chronic airflow limitation. The St. George's Respiratory Questionnaire. Am Rev Respir Dis 1992; 145: 1321-1327.

8 Goddard PR, Nicholson EM, Laszlo G, et al. Computed tomography in pulmonary emphysema. Clin Radiol 1982; 33: 379-387.

9 Prentice RL, Williams BJ, Peterson AV. On the regression analysis of multivariate failure time data. Biometrika 1981; 68: 373-379.

10 Calverley PM, Anderson JA, Celli B, et al. Salmeterol and fluticasone propionate and survival in chronic obstructive pulmonary disease. N Engl J Med 2007; 356: 775-789.

11 Tashkin DP, Celli B, Senn S, et al. A 4-year trial of tiotropium in chronic obstructive pulmonary disease. $N$ Engl $J$ Med 2008; 359: 1543-1554.

12 Vestbo J, Edwards LD, Scanlon PD, et al. Changes in forced expiratory volume in 1 second over time in COPD. N Engl J Med 2011; 365: 1184-1192.

13 Donaldson GC, Seemungal TA, Patel IS, et al. Longitudinal changes in the nature, severity and frequency of COPD exacerbations. Eur Respir J 2003; 22: 931-936.

14 de Oca MM, Tálamo C, Halbert RJ, et al. Frequency of self-reported COPD exacerbation and airflow obstruction in five Latin American cities: the Proyecto Latinoamericano de Investigation en Obstruccion Pulmonar (PLATINO) study. Chest 2009; 136: 71-78.

15 Fukuchi Y, Fernandez L, Kuo HP, et al. Efficacy of tiotropium in COPD patients from Asia: a subgroup analysis from the UPLIFT trial. Respirology 2011; 16: 825-835.

16 Tanabe N, Muro S, Hirai T, et al. Impact of exacerbations on emphysema progression in chronic obstructive pulmonary disease. Am J Respir Crit Care Med 2011; 183: 1653-1659.

17 Kim V, Criner GJ. Chronic bronchitis and chronic obstructive pulmonary disease. Am J Respir Crit Care Med 2013; 187: 228-237.

18 Jenkins CR, Celli B, Anderson JA, et al. Seasonality and determinants of moderate and severe COPD exacerbations in the TORCH study. Eur Respir J 2012; 39: 38-45.

19 Donaldson GC, Goldring JJ, Wedzicha JA. Influence of season on exacerbation characteristics in patients with COPD. Chest 2012; 141: 94-100.

20 Seemungal T, Harper-Owen R, Bhowmik A, et al. Respiratory viruses, symptoms, and inflammatory markers in acute exacerbations and stable chronic obstructive pulmonary disease. Am J Respir Crit Care Med 2001; 164: $1618-1623$.

21 Kanner RE, Anthonisen NR, Connett JE. Lower respiratory illnesses promote FEV(1) decline in current smokers but not ex-smokers with mild chronic obstructive pulmonary disease: results from the lung health study. Am J Respir Crit Care Med 2001; 164: 358-364.

22 Donaldson GC, Seemungal TA, Bhowmik A, et al. Relationship between exacerbation frequency and lung function decline in chronic obstructive pulmonary disease. Thorax 2002; 57: 847-852.

23 Wedzicha JA, Decramer M, Seemungal TA. The role of bronchodilator treatment in the prevention of exacerbations of COPD. Eur Respir J 2012; 40: 1545-1554.

24 Landbo C, Prescott E, Lange P, et al. Prognostic value of nutritional status in chronic obstructive pulmonary disease. Am J Respir Crit Care Med 1999; 160: 1856-1861.

25 Lainscak M, von Haehling S, Doehner W, et al. Body mass index and prognosis in patients hospitalized with acute exacerbation of chronic obstructive pulmonary disease. J Cachexia Sarcopenia Muscle 2011; 2: 81-86.

26 Quint JK, Baghai-Ravary R, Donaldson GC, et al. Relationship between depression and exacerbations in COPD. Eur Respir J 2008; 32: 53-60.

$27 \mathrm{Xu} \mathrm{W}$, Collet JP, Shapiro S, et al. Independent effect of depression and anxiety on chronic obstructive pulmonary disease exacerbations and hospitalizations. Am J Respir Crit Care Med 2008; 178: 913-920. 